\title{
ADVANCES IN NONLINEAR FINITE ELEMENT ANALYSIS OF AUTOMOBILES
}

\author{
K. J. Bathe†, O. Guillerminł‡, J. Walczak $\ddagger$ and Heng-Yee Chen§ \\ †Massachusetts Institute of Technology, Cambridge, MA, U.S.A. \\ †ADINA R \& D, Inc., Watertown, MA, U.S.A. \\ $\S$ Ford Motor Company, Dearborn, MI, U.S.A.
}

\begin{abstract}
The objective in this paper is to present some recent advances for the finite element analysis of automobile structures. These advances pertain to the development of effective basic finite element procedures and the efficient solution of large systems of equations. With these new procedures, it is possible to model much more accurately (and reliably) the actual nonlinear physical behavior of complex structures. The illustrative solutions given in the paper include the solution of a roof-crush problem that was solved appropriately, according to the actual physical situation, using implicit time integration (resulting practically in a static analysis). (C) 1997 Elsevier Science Ltd.
\end{abstract}

\section{INTRODUCTION}

Finite element analysis procedures are now used abundantly in the automotive industries. Linear static and dynamic analyses are conducted in a routine manner and highly nonlinear analyses are also carried out with success. However, major advances are still in great need for certain linear and for complex nonlinear analyses.

Considering linear analysis, one major concern is the efficient connection between CAD data, as created and used by the designer, and the finite element analysis process; a second concern is the creation of an appropriate mathematical model; and a third concern relates to the quality of the finite element solution of that model. Each of these areas requires significant further research and development efforts.

Considering nonlinear analysis, the same advances are required and, in addition, important basic developments in nonlinear solution techniques need be sought. While, of course, these developments represent ongoing research efforts, major advances have already been achieved in the recent years.

The objective in this paper is to review some developments that we have undertaken to enhance the possibilities for nonlinear analysis of automobiles. These developments fall essentially into two categories: namely, firstly, we have developed various more-effective basic finite element procedures and, secondly, we have enhanced the solution of large systems of equations. These ingredients in the ADINA analysis system render it possible to solve, in a reliable manner, more complex problems than ever before.

In the following sections, we first briefly describe our advances in basic finite element analysis procedures. These advances pertain to the reliable and effective solution of large strain-incompressible and elasto-plastic response, the large deformation analysis of shells, and the analysis of contact problems. We then focus on our developments for the solution of large systems of equations and the need for using static analysis or implicit dynamic analysis procedures when a slow process (such as roof crush) is to be analyzed. Explicit analysis procedures should not be employed for such solutions (although these are being used in practice because the implicit codes employed cannot solve for the slow motion response). In Section 4, we then present solution results in order to underline some of our theoretical observations and in order to demonstrate our advances in the field. We conclude in Section 5 that our new capabilities have significantly advanced the field. However, of course, important further enhancements are still very much sought.

\section{ADVANCES IN BASIC FINITE ELEMENT PROCEDURES}

For automotive nonlinear structural analysis, basic finite element procedures in three areas are of great importance and we have made significant progress in each of these fields: the analysis of (almost) incompressible media; the large deformation analysis of shells including composite shells and plasticity; and the analysis of contact conditions. In the following sections, we briefly describe our advances in each of these areas.

\subsection{Analysis of (almost) incompressible media}

The analysis of (almost) incompressible media, such as rubber-like materials, or metals in elasto-plasticity or creep, in plane strain, axisymmetric or three-dimensional conditions, should only be performed using elements that satisfy the ellipticity and 
inf-sup conditions [1]. If these conditions are satisfied, the finite element discretization is stable and optimal. The usually-effective pure displacementbased finite element discretizations do not satisfy the inf-sup condition and a mixed method must be employed. Very powerful discretization procedures are based on displacement-pressure interpolations, which, when the appropriate displacement and pressure interpolations are employed, satisfy the ellipticity and inf-sup conditions.

Let $V_{h}$ be the space of displacement interpolations, $Q_{h}$ be the space of pressure interpolation, and $K_{h}$ be given by:

$$
K_{h}=\left\{\mathbf{v}_{h} \mid \mathbf{v}_{h} \in V_{h}, \operatorname{div} \mathbf{v}_{h}=0\right\}
$$

Then the ellipticity condition is:

$$
a\left(\mathbf{v}_{h}, \mathbf{v}_{h}\right) \geq \alpha\left\|\mathbf{v}_{h}\right\|^{2} \forall \mathbf{v}_{h} \in K_{h}
$$

where $a(.,$.$) is the bilinear form corresponding to the$ deviatoric strain energy and $\alpha$ is a constant, greater than zero and independent of $h$. The inf-sup condition is:

$$
\inf _{q_{h} \in Q_{h}} \sup _{V_{h} \in V_{h}} \frac{\left(\int_{V o l} q_{h} \operatorname{div} V_{h} \mathrm{~d} V o l\right)}{\left\|q_{h}\right\| V_{h} \|} \geq \beta>0
$$

where $\beta$ is a constant independent of $h$.

These conditions are crucial to be satisfied for (almost and totally) incompressible analysis and are discussed at length in Ref. [1]. In addition, various elements are summarized in that reference which satisfy these conditions and provide reliable and effective discretization schemes. Simply using "a reduced integration' scheme on a displacement-based formulation that has not been analyzed as a mixed method and proven to be stable and convergent is certainly quite inadequate.

\subsection{Large deformation analysis of shells}

The key ingredient in models for the large deformation analysis of thin shell structures is the finite elements used. As in the area of incompressible analysis, pure displacement-based elements are not effective and a mixed formulation must be used. We have developed the MITC shell elements which are effective for thick and thin shell structures [1,2]. However, another required task for a successful shell analysis is the generation of a 'valid' mesh. In the automotive industries, geometrically complex thin shell structures need to be meshed and a valid mesh should satisfy the following requirements:

- the mesh should be properly connected in all its parts; that is, unconnected regions or regions that can undergo rigid body motions are inadmissible;
- for essentially flat or smoothly curved parts of the shell only the five natural nodal degrees of freedom should be assigned, whereas for intersections, six nodal degrees of freedom need to be used.

If the ADINA-IN pre-processor is employed, a valid mesh can be directly constructed using the automatic generation procedures available in the program. Therefore, in a linear analysis, no zero pivot elements will be encountered in the Gauss elimination solution, and in a nonlinear analysis reliable results are obtained.

We should note that if the above requirements are not fulfilled in a linear analysis, the zero (or, due to round-off errors, very small) pivot elements may be simply interpreted to correspond to nodal degrees of freedom with no stiffness (after static condensation of the preceding degrees of freedom) which do not affect the response of the rest of the model. However, in nonlinear analysis, such degrees of freedom cannot be admitted because zero pivots shall correspond only to true physical instabilities.

There is, in addition, another geometric requirement that needs to be fulfilled in nonlinear analysis (and strictly also in a linear analysis):

- the shell structure should not, in its initial configuration, unphysically penetrate itself or other media.

A linear analysis will, in general, not detect such penetrations, but a nonlinear analysis that automatically searches for and includes contact conditions will detect them. A nonlinear solution is then still possible if the physical overlaps and interpenetrations are reasonable. However, they are frequently due to modeling errors and provide physically unrealistic conditions, in which case the nonlinear solution should be terminated. (Of course, the nonlinear analysis could simply be continued by ignoring these conditions, but then unrealistic results are generated.)

Hence, it is clear that for nonlinear analysis, the finite element model must be established in a much more stringent manner than if only a linear analysis is to be pursued.

\subsection{Analysis of contact}

In nonlinear analysis of automobile structures, contact conditions are frequently an important phenomenon to be included. Let $g$ be the gap between two contacting media (for example, two thin shell components) and $\lambda$ be the magnitude of the normal component of the contact traction (compression being positive). Then the conditions for normal contact are:

$$
g \geq 0 ; \lambda \geq 0 ; g \hat{\lambda}=0
$$


where the last equation expresses the fact that the gap must be zero when $\lambda$ is greater than zero, and vice versa.

Let $t$ be the magnitude of the tangential component of the contact traction and $\dot{u}$ be the magnitude of the relative tangential velocity between the contacting media at the point considered; then, using Coulomb's law of friction, the tangential conditions during contact are (with $\lambda>0$ )

$$
\begin{aligned}
& \left|\frac{t}{\mu \lambda}\right| \leq 1,\left|\frac{t}{\mu \lambda}\right|<1 \text { implies } \dot{u}=0, \\
& \left|\frac{t}{\mu \lambda}\right|=1 \text { implies } \dot{u} \neq 0 .
\end{aligned}
$$

The solution of media in contact conditions therefore entails the usual equations of analysis of the media plus the satisfaction of eqns (4) and (5).
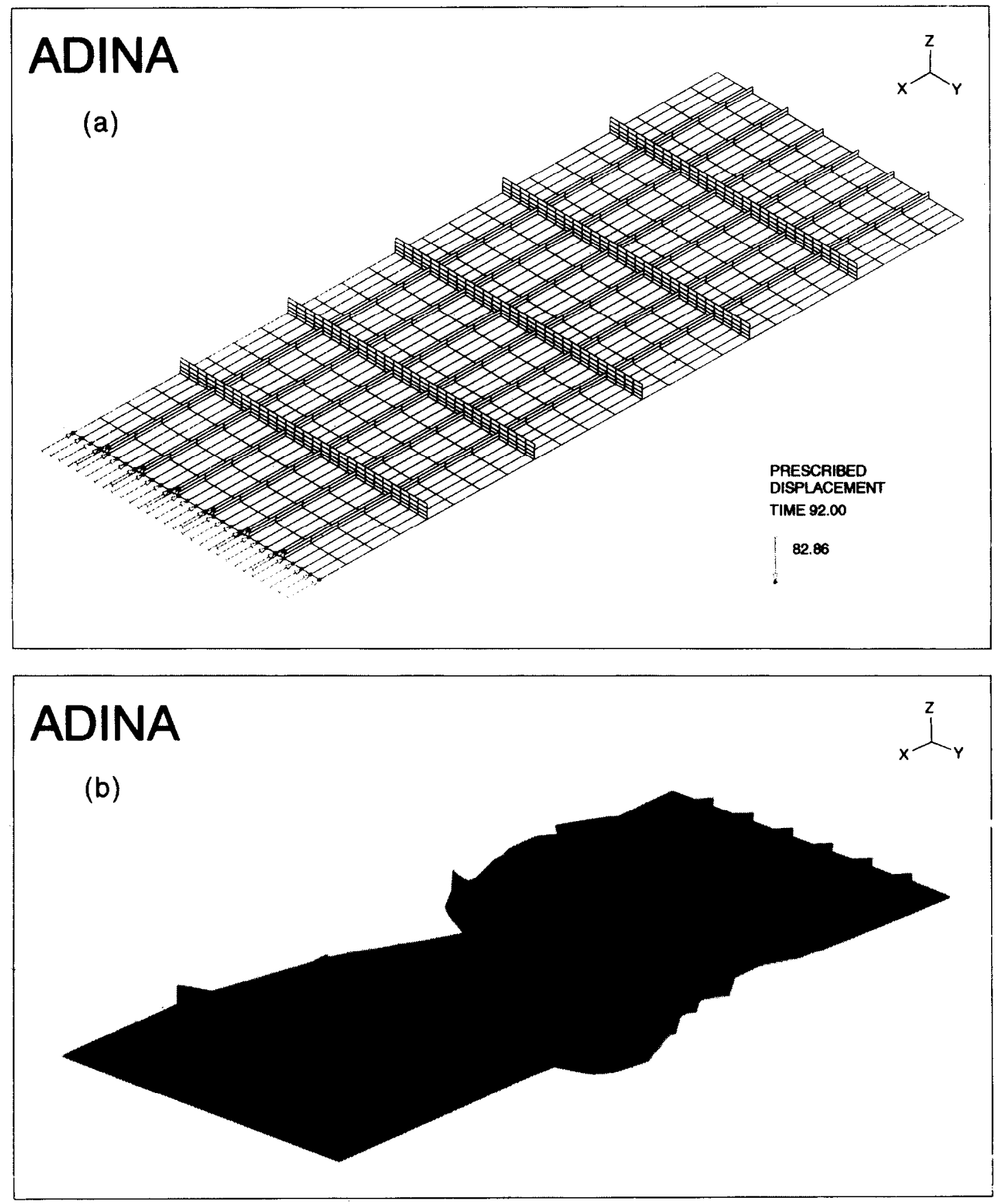

Fig. 1. Analysis of stiffened panel: (a) mesh used; (b) collapsed state at imposed displacement of $1.5 \mathrm{~m}$. 
We have developed, in ADINA, the constraint function algorithm $[1,3]$, which can be used to solve very complex contact conditions, including contact of a surface onto itself. The solution algorithm has been developed for static analysis or dynamic solutions based on implicit time integration. A key ingredient is that continuous and differentiable (constraint) functions for any values of $g, \lambda, \dot{u}$ and $t / \mu \lambda$ are used to enforce the conditions of eqns (4) and (5). In our implementation, we employ a Lagrange multiplier method to impose the constraint functions but, of course, a penalty method could be used as well [1]. Because of the continuity of the functions, derivatives can be taken to obtain a Jacobian that can be incorporated into the stiffness matrix of the problem [1]. However, full quadratic convergence is frequently not obtained, because the models of the contact surfaces are not sufficiently smooth.

\section{SOLUTION OF LARGE SYSTEMS OF EQUATIONS}

The finite element analysis process consists of solving the governing equations of a mathematical model pertaining to the physical situation [1]. If the physical process corresponds to a static situation, then clearly the mathematical model should be a static model, while if the physical process corresponds to a dynamic situation (structural vibration or wave propagation) then the mathematical model should be a dynamic model. In other words, the actual physical situation should be accurately represented in the mathematical model which is solved using finite element procedures.
When solving mathematical models in the automotive industries, large finite element systems frequently need to be considered. If explicit time integration is applicable and used, very large finite element systems can be solved without great difficulties. However, a static or implicit time integration dynamic solution can represent a significant task if complex nonlinearities are present. Indeed, in many cases, finite element programs cannot be used to solve for the static response because convergence is not reached in the iterations. Such a solution is then abandoned and a dynamic solution based on explicit time integration is pursued [4]. This dynamic solution is based on a simple forward integration without iteration and is much easier to obtain.

However, explicit time integration is really designed for dynamic wave propagation problems and an integration time step which is smaller than or equal to the critical time step needs to be employed. To use explicit time integration in a reasonably effective manner for an essentially static physical situation means, in fact, that the mathematical model must be changed. The calculated data may then have little resemblance to the actual physical response sought. Also, valuable analysis information that is calculated in a static analysis, such as bifurcation points, cannot be obtained when using explicit time integration (see Section 4.1).

During recent years, significant improvements in the nonlinear finite element procedures for contact conditions, large deformations, and elasto-plasticity have been accomplished (see Section 2) and, in addition, iterative solvers, sparse matrix solvers and parallel processing techniques have been developed.

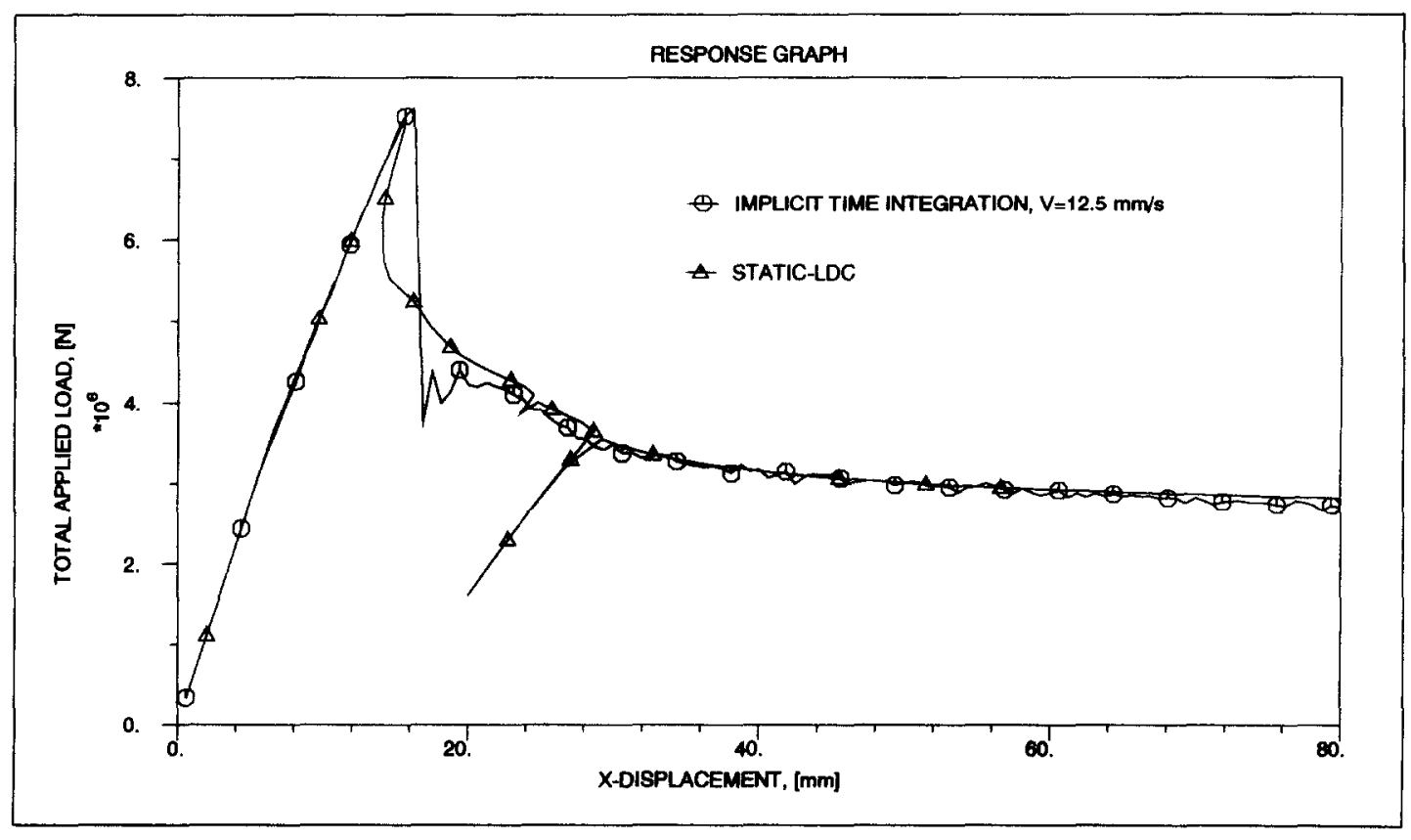

Fig. 2. Load-displacement curve of stiffened panel; static analysis and implicit dynamic results. The static solution has been obtained with the load-displacement-constraint (LDC) method. 


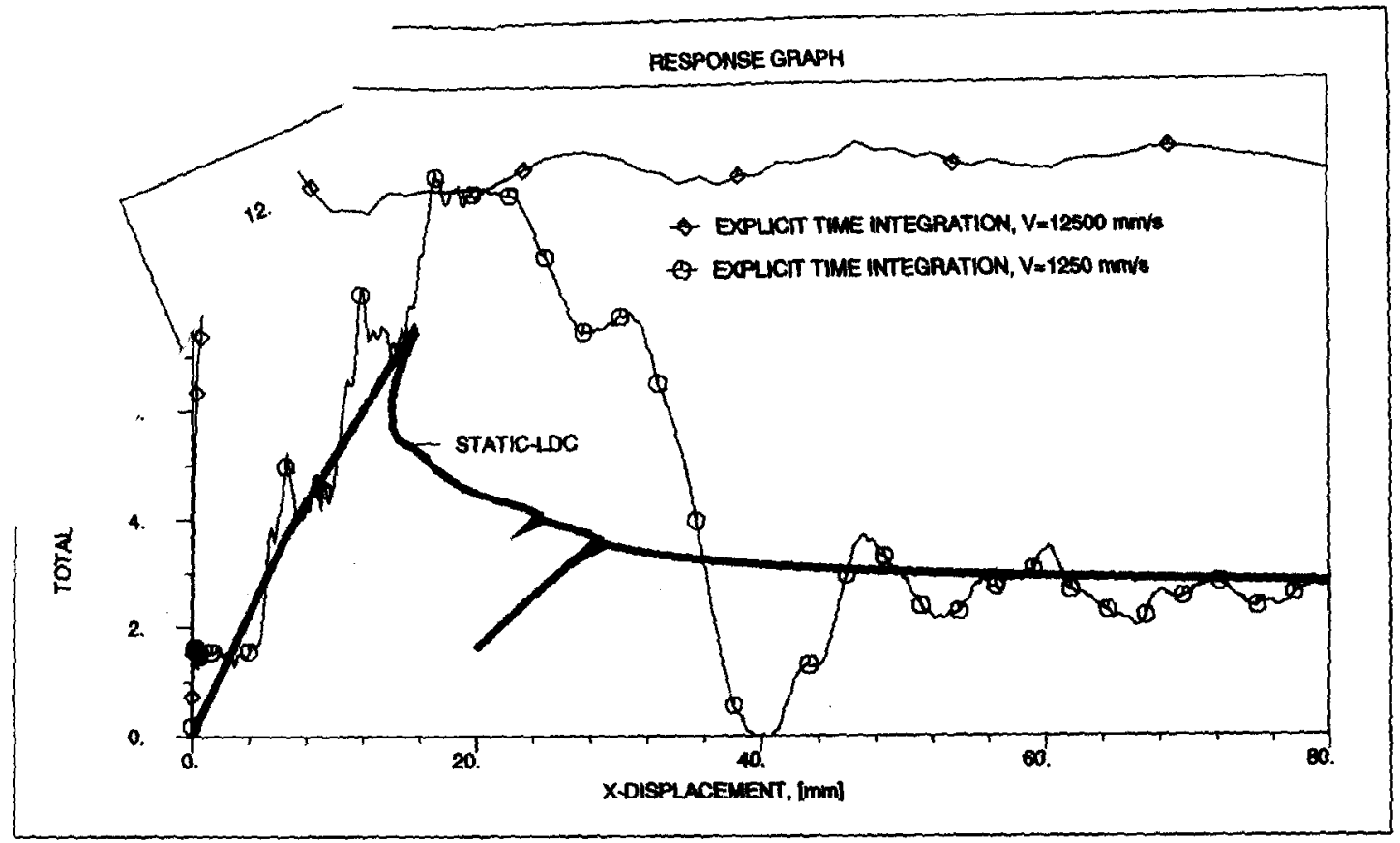

Fig. 3. Response curves calculated in analysis of stiffened panel using explicit time integration assuming larger speeds of displacement application.

We have reported earlier on the capabilities of the iterative solvers in the ADINA system [5]. The sparse solvers and parallel processing techniques have been implemented since that time and represent further significant advances. The sparse solvers are available for the solution of the simultaneous equations in static and implicit dynamic analyses and for frequency solutions. This solution approach is particularly effective for the analysis of shell structures as encountered in the automotive industries, because iterative techniques are then frequently not sufficiently effective due to the bad conditioning of the coefficient matrix.

Considering the parallel processing in ADINA, the procedures have been implemented for shared memory machines and for all phases in the analysis:

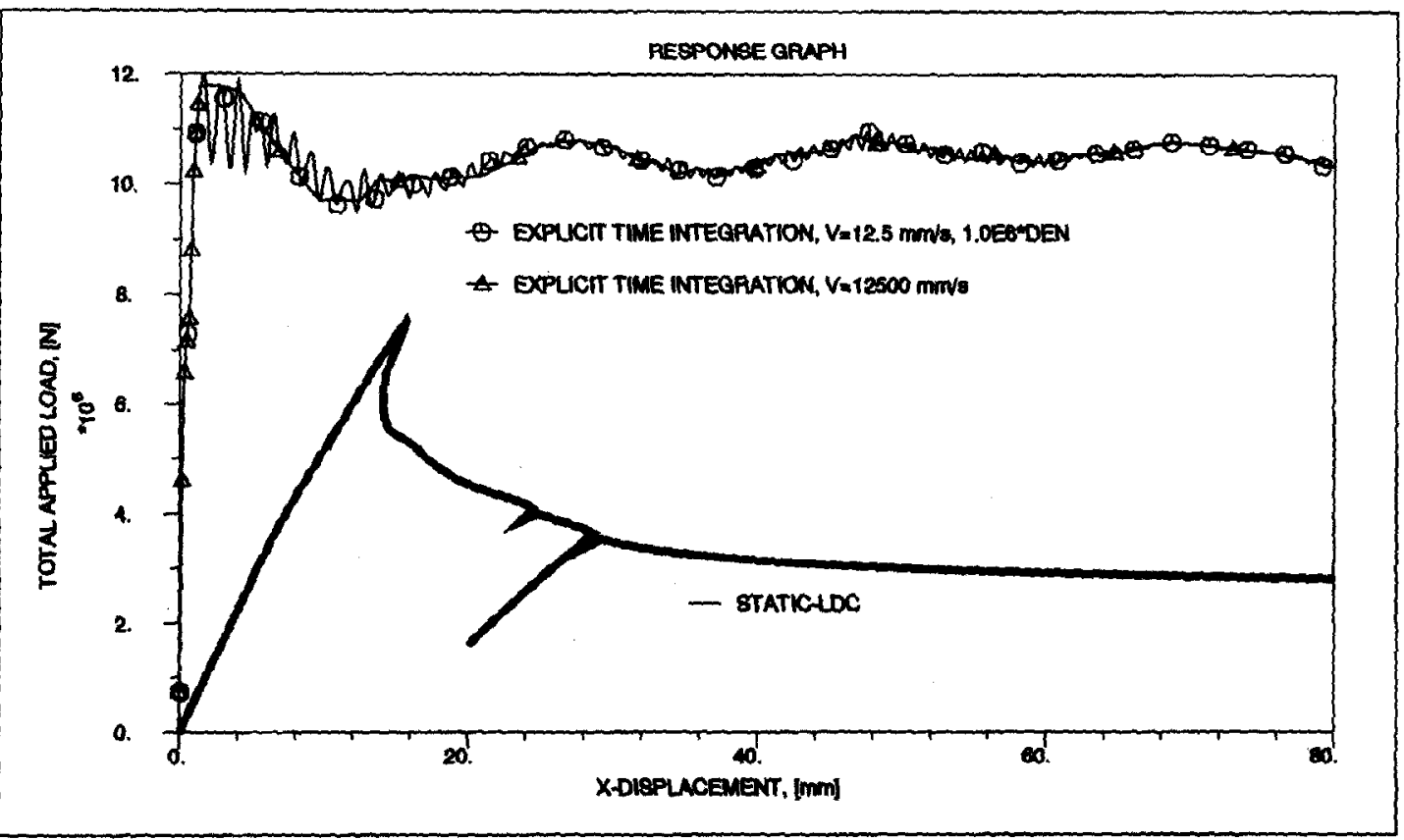

Fig. 4. Response curve using explicit time integration assuming artificially high mass density. 


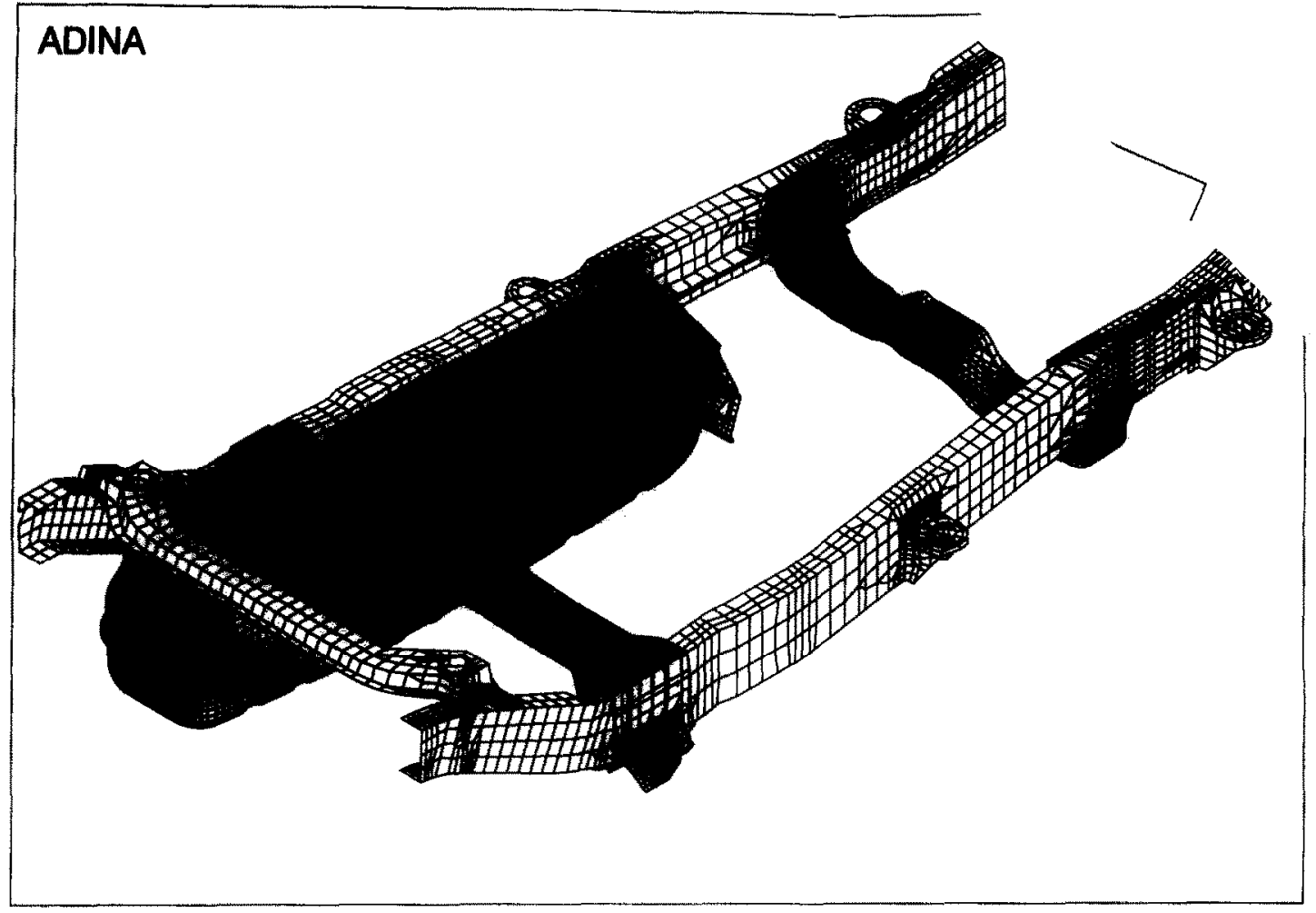

Fig. 5. Shell model of fuel tank.

the element stiffness calculations, the assemblage of the element matrices and the equation solution. Together, the sparse matrix and parallel processing technologies can decrease the solution times drastically when solving large systems. Since significant improvements in the convergence characteristics of the nonlinear finite element procedures have also been obtained (see Section 2), it is now frequently possible to perform, in an effective manner, static analyses or dynamic analyses based on implicit time integration as dictated by the actual physics of the problem. There is no need to forcibly use explicit timc integration. We demonstrate these achievements in the next section.

\section{ANALYSIS CASES}

We present in this section the results of some analyses that underline our theoretical observations and illustrate the analysis capabilities. We refer the reader also to $\operatorname{Refs}[1,3]$.

\subsection{Analysis of stiffened panel}

Figure 1 shows the model of the stiffened panel considered. (This is actually a panel of a ship structure.) The steel panel has a dimension of $4.20 \times 10.81 \mathrm{~m}$ and a thickness of $0.006 \mathrm{~m}$ and is simply-supported along all edges. The model consists of four-node shell elements, which can represent the large deflection and elastic-plastic behavior. A collapse analysis of the panel is sought when the $x$-displacement at one end is prescribed slowly, namely at $12.5 \mathrm{~mm} \mathrm{~s}^{-1}$. We present this example analysis to illustrate that a static analysis, for the physics considered, provides (of course) the appropriate solution and, in addition, is also computationally more effective than an accurate explicit dynamic solution.

Figure 2 shows the response calculated statically. A characteristic of the load carrying capacity is the sudden and very significant decrease in load at about $16 \mathrm{~mm}$ displacement. There is also a bifurcation at about $28 \mathrm{~mm}$, which may, however, not be significant in practice. A total of 90 incremental steps were carried out for the static analysis to $80 \mathrm{~mm}$. Figure 1(b) shows the static results at a prescribed displacement of $1.5 \mathrm{~m}$, just to indicate that the static solution could be obtained with ADINA to very large deformations.

Figure 2 also shows the response calculated in an implicit dynamic analysis when applying the actual velocity of $12.5 \mathrm{~mm} \mathrm{~s}^{-1}$. The time step $\Delta t$ was $0.05 \mathrm{~s}$ and a total of 128 steps were used. We note that the calculated response is very close to the response obtained statically.

Table 1. Solution times in analysis of fuel tank. Computer used: SGI Power Challenge; no. of eqns $=154,186$

\begin{tabular}{cccc}
\hline \multicolumn{4}{c}{ Solution time per stcp (10 steps total) } \\
\hline $11 \mathrm{~min}$ & $7 \mathrm{~min} 18 \mathrm{~s}$ & $4 \mathrm{~min} 28 \mathrm{~s}$ & $3 \mathrm{~min} 54 \mathrm{~s}$ \\
1 processor & 2 processors & 4 processors & 6 processors \\
\hline
\end{tabular}




\section{ADINA}

$\pi N=0.0$

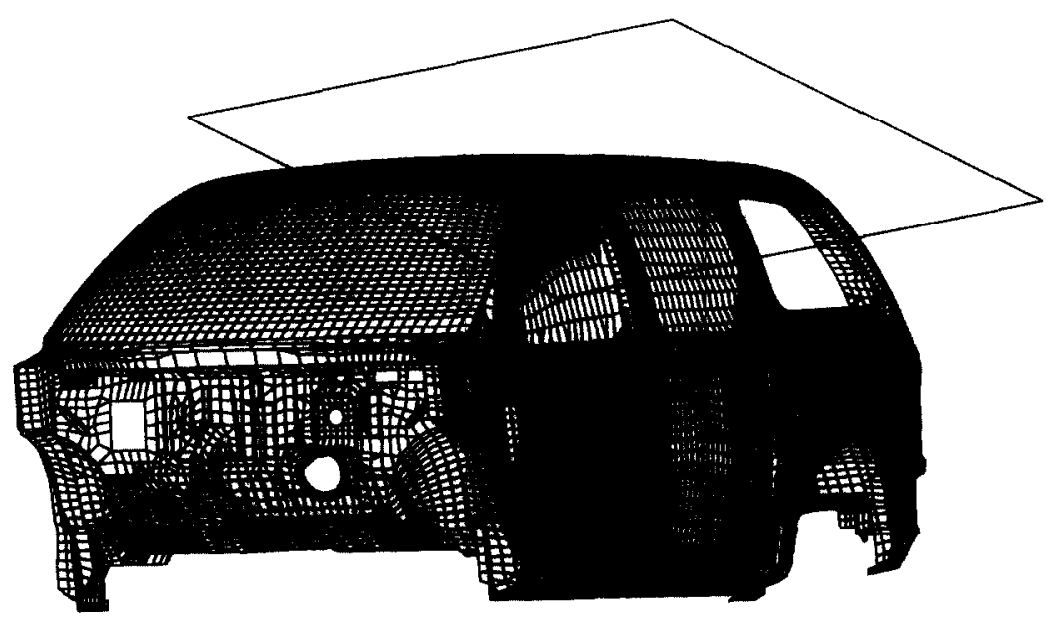

Fig. 6. Finite element shell model of car, also showing the rectangular rigid contact surface causing crushing. 

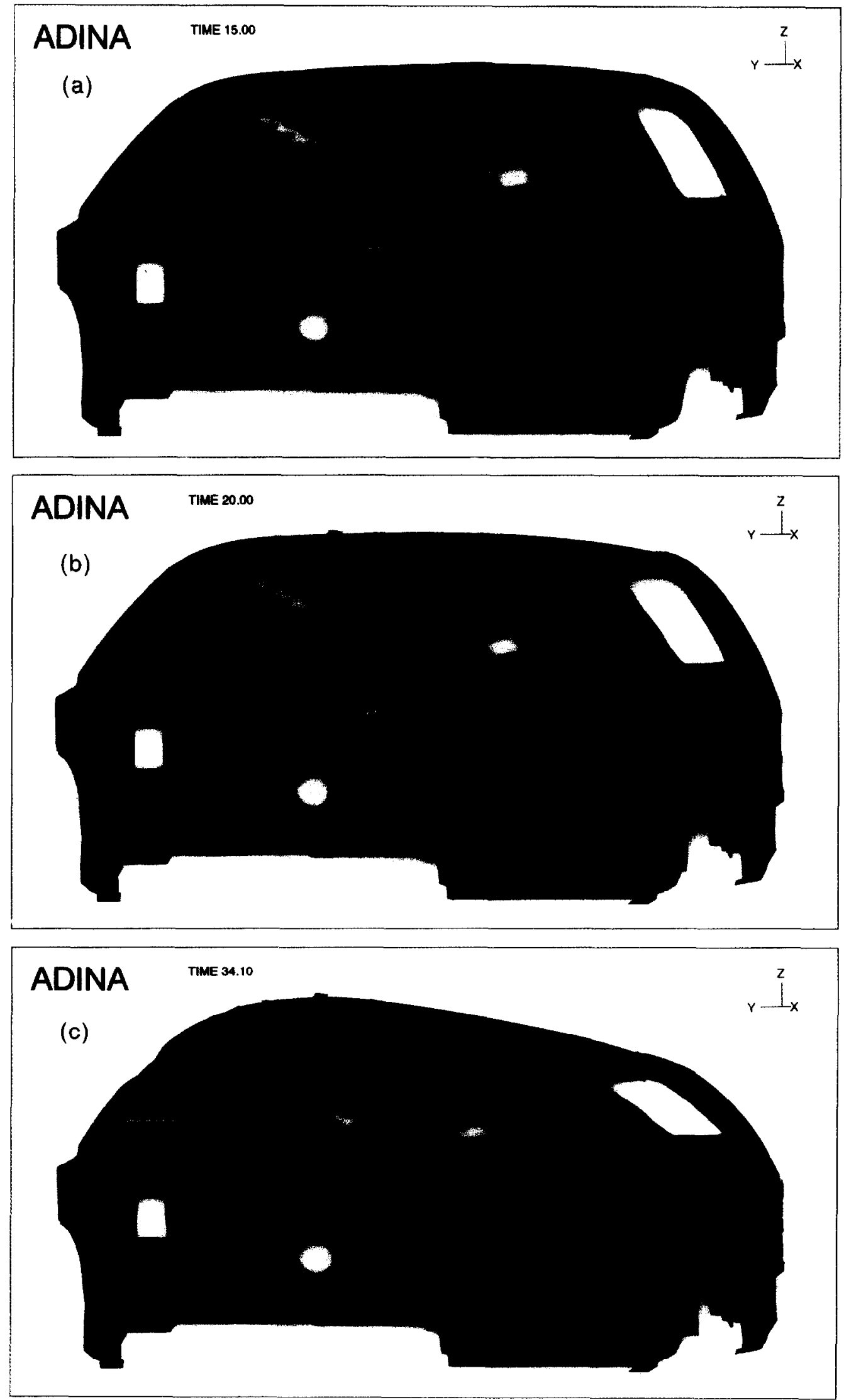

Fig. 7. Crushed configurations of the car model at different levels of deformations, to about 18 in of crush deflection. 


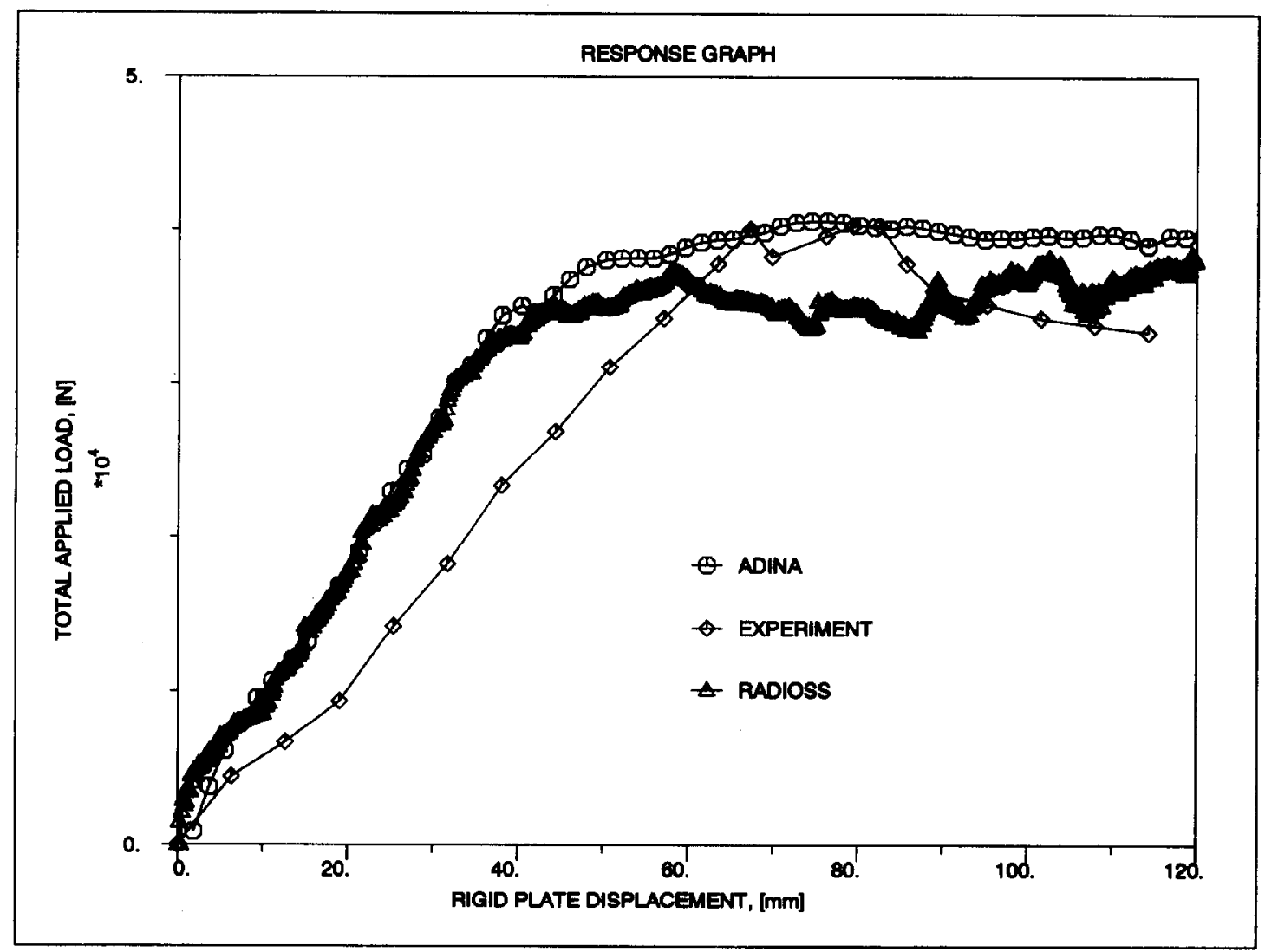

Fig. 8. Load-displacement curves for car. Experimental and ADINA results.

For an explicit time integration solution, the (approximate) critical time step size is $\Delta t_{\text {crit }}=10^{-6} \mathrm{~s}$ and, hence, for a velocity of $12.5 \mathrm{~mm} \mathrm{~s}^{-1}$, about $6,400,000$ steps would need to be used to calculate the response to $80 \mathrm{~mm}$. This would require too-large computational resources and, hence, we calculated the response when the velocity of the prescribed displacement application was $1250 \mathrm{~mm} \mathrm{~s}^{-1}$ and $12,500 \mathrm{~mm} \mathrm{~s}^{-1}$. The number of time steps was 64,000 and 6400 , respectively. Figure 3 shows the calculated response. It is seen that the predicted response is very different from the actual required physical response (corresponding to $12.5 \mathrm{~mm} \mathrm{~s}^{-1}$ ).

Regarding the solution times used, we note that the static and implicit dynamic solutions and the 6400 steps explicit dynamic solution did not require a significantly different solution effort, but the explicit dynamic solution using 64,000 steps, which is still highly inaccurate, was much more expensive.

Figure 4 shows the response calculated when we increased the mass density (quite artificially) by a factor of $10^{6}$, so that the critical time step size increased by a factor of $10^{3}$. Of course, such an increase in mass density is quite inappropriate and the calculated response is again far from the response sought (but is close to the response obtained with the increase in velocity of the prescribed displacement to $v=12500 \mathrm{~mm} \mathrm{~s}^{-1}$ ). Clearly, this study shows that the explicit solution is quite inappropriate for this problem.

\subsection{Analysis of fuel tank}

Figure 5 shows the fuel tank shell model considered. The model was generated by translating, and adding to, a NASTRAN input deck of shell elements for an ADINA nonlinear analysis. The ADINA model consists of MITC4 shell elements, includes contact conditions, large deformations and plasticity, and comprises over 150,000 equations. The model was loaded statically with an acceleration of $10 \mathrm{~g}$ vertically.

We include this example analysis here because we wish to show that large nonlinear analyses can now be performed quite effectively. The solution of this model was obtained in 10 incremental steps, with Newton-Raphson iterations in each step. An SGI Power Challenge machine was employed and Table 1 summarizes the solution times used for the model depending on the number of processors employed. We see that using four processors, the complete nonlinear analysis is obtained in less than one hour of solution time.

\subsection{Analysis of roof-crush problem}

The motor-car shell model shown in Fig. 6 is a RADIOSS (an explicit code) model that we have also 
run using ADINA. The sheet metal parts are modeled with single layered MITC4 shell elements.

The windshield is modeled with multilayered MITC4 shell elements using a glass-PVB-glass stacking, which can fracture. The joint between the windshield and metal frame and a large number of spot welds are modeled using spring elements. Hinges and locks are modeled with constraint equations. The bottom of the car representation is fixed in all displacements.

The model was loaded with a rigid plane at its left corner slowly (at a speed of $0.05 \mathrm{~km} \mathrm{~h}^{-1}$ ) crushing the motor car shell. The analysis represents an actual laboratory test, where we assume that the testing device can be represented as a rigid surface submitted to prescribed displacements. In the analysis, contact between the car body and testing device and between the car parts themselves using a self-contact option is taken into account. In the sheet metal parts, large deformations and plasticity are included.

The complete model consists of 296,273 equations that were solved incrementally using a full NewtonRaphson iteration in each step. Implicit time integration was used, but because the speed of load application (crushing by the rigid plane) was very slow, the analysis corresponds to an almost static solution. The test required crush deflection of $180 \mathrm{~mm}$ (about $7 \mathrm{in}$ ) was reached using the automatic time stepping algorithm in ADINA in about $24 \mathrm{~h}$ of solution time on a Power Challenge machine (with four processors). Figure 7 shows some deformed configurations of the shell model.

Figure 8 gives the ADINA calculated force--deflection results and compares these with experimental results and an explicit time integration solution reported using the RADIOSS program. We can make some interesting observations regarding these results. Note that the experimental results give a stiffness prior to collapse (slope of the curve at displacements smaller than the collapse displacements) that is substantially smaller than the stiffness calculated using ADINA and RADIOSS. The explanation for this difference is that the finite element model solved does not include the complete right side of the structure. Instead of including this part of the structure, the model was fixed at the level of the doors, making the model substantially stiffer.

On the other hand, the collapse loads calculated in the finite element solutions are quite close to the experimental result. The reason for this good match is that the finite element model was tuned by the analysts using RADIOSS to obtain this good comparison; for example, the windshield was not fully bonded to the frame around its complete perimeter. The fact that the ADINA solution of the RADIOSS finite element model is even closer to the experimental collapse load than the RADIOSS solution is, perhaps, just fortuitous.
In this analysis, the reported RADIOSS explicit and the ADINA implicit solution results are not very different. However, we refer to the analysis results shown in Figs $1-4$ to emphasize that explicit time integration must be used with great care in the analysis of slow dynamic (i.e. almost static) response calculations, for example, when local or global post-buckling phenomena with decreases in load carrying capacity are present. Such decreases in load carrying capacity may be totally missed in explicit time integration leading to an unconservative design. This consideration is particularly important when seeking an optimum design, because relatively small changes in geometry, thickness and boundary conditions may result in significant response differences [6].

This solution illustrates that large models in the automobile industries can now be solved according to the actual physical situation-here, an almost static solution was obtained as dictated by the physics. There is no need to solve this type of problem using explicit time integration, which generally requires a change in the physical situation to allow a reasonably effective solution and can lead to missing important physical phenomena in the analysis.

\section{CONCLUSIONS}

The objective in this paper was to present some recent advances in finite element capabilities for the analysis of complex automotive structures. These advances are significant and pertain to various basic finite element procedures-namely, an effective contact algorithm, powerful shell elements and plasticity solution algorithms - and pertain to new equation solving procedures and parallel processing. For an effective advanced nonlinear analysis, all these capabilities are important and must work efficiently together. With these capabilities, it is now possible to solve complex physical situations significantly more effectively and also more reliably than is frequently recognized in practice. In particular, implicit dynamic or static solutions can be obtained for a physical situation of slow motion when, previously in other authors' works, the analysis conditions were changed artificially to obtain an explicit dynamic solution. We demonstrated in this paper that such an explicit solution can be significantly in error, and also computationally more expensive than the appropriate static or implicit dynamic solution.

\section{REFERENCES}

1. Bathe, K. J., Finite Element Procedures. Prentice Hall, Englewood Cliffs, NJ, 1996.

2. Bathe, K. J. and Dvorkin, E. N., A formulation of general shell elements-the use of mixed interpolation of tensorial components. International Journal for Numerical Methods in Engineering, 1986, 22, 697-722. 
3. Bathe, K. J. and Bouzinov, P. A., On the constraint function method for contact problems. Computers and Structures, 1997, 64, 1069-1085.

4. Makinouchi, A., Nakamachi, E., Oñate, E. and Wagoner, R. H. (eds), In Proceedings, NUMISHEET '93, Isehara, Japan, 1993.
5. Bathe, K. J., Walczak, J. and Zhang, H., Some recent advances for practical finite element analysis. Computers and Structures, 1993, 47/5, 511-521.

6. Chapelle, D. and Bathe, K. J., Fundamental considerations for the finite element analysis of shell structures. Computers and Structures, in press. 